\title{
Procalcitonin as a diagnostic marker of ventilator-associated pneumonia in cardiac surgery patients
}

\author{
JIA JIAO $^{1,2}$, MIN WANG $^{1}$, JIANFENG ZHANG $^{1}$, KANGJUN SHEN $^{1}$, XIAOBO LIAO $^{1}$ and XINMIN ZHOU ${ }^{1}$ \\ ${ }^{1}$ Department of Cardiothoracic Surgery, The Second Xiangya Hospital, Central South University, Changsha, \\ Hunan 410011; ${ }^{2}$ Department of Cardiothoracic Surgery, The First Affiliated Hospital of Chongqing Medical University, \\ Chongqing 400016, P.R. China
}

Received May 20, 2014; Accepted December 19, 2014

DOI: 10.3892/etm.2015.2175

\begin{abstract}
The aim of the present study was to assess whether procalcitonin (PCT) can be used as a diagnostic marker for ventilator-associated pneumonia (VAP) in cardiac surgery patients. Between January 2012 and June 2013, a total of 92 patients were recruited and divided into non-VAP (59 patients) and VAP (33 patients) groups. The preoperative and postoperative characteristics of the patients were recorded. Serum levels of PCT, interleukin (IL)-6 and C-reactive protein (CRP) were measured using an electrochemiluminescence immunoassay. Subsequently, receiver operating characteristic curves of the PCT, IL-6 and CRP levels were constructed. In addition, associations between the sequential organ failure assessment (SOFA) scores and the serum levels of PCT, IL-6 and CRP in the VAP patients were analyzed. No statistically significant difference was observed between the non-VAP and VAP patients in the occurrence of postoperative complications. However, the SOFA scores (days 1 and 7), the duration of stay in the intensive care unit and the mechanical ventilation time were all significantly higher in the VAP group when compared with the non-VAP group $(\mathrm{P}<0.05)$. The optimum PCT cut-off value for VAP diagnosis on day 1 was $5.0 \mathrm{ng} / \mathrm{ml}$, with a sensitivity of $91 \%$ and a specificity of $71 \%$. The serum PCT levels on days 1 and 7 were found to correlate positively with the SOFA scores $(r=0.54$ and $r=0.66$ for days 1 and 7 , respectively). Therefore, the results suggested that serum PCT may be used as diagnostic marker for VAP in patients following cardiac surgery.
\end{abstract}

\section{Introduction}

Major cardiac surgery patients are a particularly high-risk population for nosocomial infections (1). Ventilator-associated

Correspondence to: Dr Xinmin Zhou, Department of Cardiothoracic Surgery, The Second Xiangya Hospital, Central South University, 139 Middle Renmin Road, Changsha, Hunan 410011, P.R. China E-mail: zhxinminn@163.com

Key words: procalcitonin, ventilator-associated pneumonia, cardiac surgery, diagnostic marker pneumonia (VAP) is the primary infectious complication for cardiac surgery patients (2) and is associated with a marked increase in morbidity and mortality rates (3). The prevalence of VAP following cardiac surgery is estimated to be $17.9 \%$ in patients who receive mechanical ventilation for $>48 \mathrm{~h}$ and $28.8 \%$ in patients who undergo mechanical ventilation for $>72 \mathrm{~h}$ (4). The median mechanical ventilation time and duration of hospital stay are significantly prolonged in patients who develop VAP following cardiac surgery (5). Tamayo et al determined that cardiac surgery patients who develop VAP have a mortality risk that is 8.53 times higher than those without VAP (6). In addition, the authors showed that VAP is the most important independent risk factor for mortality following major cardiac surgery. Therefore, early prevention, diagnosis and treatment of VAP is essential.

Procalcitonin (PCT), a protein of 116 amino acids with a molecular weight of $13 \mathrm{kDa}$, is the precursor molecule of calcitonin. PCT is usually produced by parafollicular cells in the thyroid gland (7). Since PCT levels were found to be elevated in patients with bacterial infection, increasing interest has been directed at the possibility of using PCT as an indicator of infection (7-9). In addition, PCT has been shown to be useful in discriminating between septic and nonseptic inflammation and may be a promising biomarker for the diagnosis of VAP (10). However, cardiac surgery and the use of cardiopulmonary bypass (CPB) may activate the immune system and lead to a systemic inflammatory response, which increases the difficulty of identifying infectious complications in cardiac surgery patients. Furthermore, CPB and noninfectious complications, such as organ dysfunction/failure, contribute to increased PCT levels (11). Therefore, the value of PCT as a diagnostic marker of infection in cardiac surgery remains controversial. Adib-Conquy et al (12) showed that PCT is not specifically a marker for infection, as PCT levels may increase markedly in cases of acute inflammation with no infection. In addition, Sinning et al (13) suggested that systemic inflammatory response syndrome (SIRS) is associated with PCT in patients with a transcatheter aortic valve implantation. Therefore, the feasibility of using PCT as a diagnostic marker for VAP in cardiac surgery patients remains unclear and requires further study.

The present study examined 92 patients who received $\geq 48 \mathrm{~h}$ of mechanical ventilation following cardiac surgery. 
The patient serum levels of PCT, interleukin (IL)- 6 and C-reactive protein (CRP) were analyzed using an electrochemiluminescence immunoassay (ECLIA). The incidence and type of postoperative noninfectious complications in the patients were also recorded. In addition, the diagnostic values of serum levels of PCT, IL-6 and CRP were analyzed by linear regression.

\section{Materials and methods}

Patients. The study was conducted at the intensive care unit (ICU) of the Department of Cardiothoracic Surgery at the Second Xiangya Hospital of Central South University (Changsha, China). In total, 92 consecutive patients (age, $\geq 18$ years) who had received $\geq 48$ h of mechanical ventilation were recruited between January 2012 and June 2013. All the patients had undergone elective cardiac surgery with CPB. Patients were excluded from the study if they had been diagnosed with pneumonia prior to the mechanical ventilation, were pregnant, had received immunosuppressants or long-term corticosteroid therapy or had a coexisting extrapulmonary infection that required antibiotic therapy for more than three days prior to or following the cardiac surgery. The patients were divided into VAP and non-VAP patient groups.

The study protocol was approved by the Ethics Committee of the Second Xiangya Hospital of Central South University, and patients provided written informed consent.

Diagnosis of VAP and classification of complications. The study focused on patients in the early stages of VAP that had been diagnosed between $48 \mathrm{~h}$ and six days after initiation of the mechanical ventilation. Diagnosis of VAP (14) was determined using a novel criteria, based on chest X-ray results, with at least two of the following features: Fever with a body temperature of $>38^{\circ} \mathrm{C}$; a white blood cell count of $>11,000$ or $<3,000 / \mu 1$; or the presence of purulent endotracheal secretions. Microbiological samples were obtained by fibroscopic bronchoalveolar lavage (BAL), and the growth of $\geq 10^{4}$ colony-forming units $/ \mathrm{ml}$ microorganism culture of BAL was considered to be positive.

A number of noninfectious complications were defined according to specific criteria. Cardiovascular complications were defined as a low cardiac output with a cardiac index of $<2.01 / \mathrm{min} / \mathrm{m}^{2}$, postoperative myocardial infarction and malignant ventricular arrhythmia (sustained and requiring further treatment). Pulmonary dysfunction was diagnosed if the $\mathrm{PaO}_{2} / \mathrm{FiO}_{2}$ ratio was $<200 \mathrm{mmHg}$, unless this was caused by cardiogenic factors. Acute renal failure was defined as requiring temporary hemofiltration or hemodialysis, while neurological complications included stroke and ischemic cerebral infarction. Finally, rethoracotomy due to bleeding was a potential noninfectious complication.

Data collection. Follow-up lasted for seven days or until patient mortality. Patient characteristics and clinical data, including the age, gender, preoperative diagnosis, left ventricular ejection fraction, brain natriuretic peptide level, type of surgery, CPB time, aortic cross-clamp time, mechanical ventilation time, length of ICU stay and diagnosis of noninfectious complications, were collected.
Biological measurements and sequential organ failure assessment (SOFA). Blood samples were collected prior to cardiac surgery in order to define the baseline biological measurement values. Patients were evaluated daily for symptoms of VAP. Once VAP was diagnosed (defined as day 1), the patients received empirical antibiotic treatment until the results of the bacterial culture were available. In the non-VAP group, day 1 was defined as the day when the tracheal intubation was removed from each patient. The levels of PCT, IL-6 and CRP were measured using an ECLIA (Roche Diagnostics $\mathrm{GmbH}$, Mannheim, Germany) for the patients in the VAP and non-VAP groups on days $1,3,5$ and 7 .

SOFA was conducted on days 1 and 7 for the patients in the VAP and non-VAP groups (15).

Statistical analysis. Data are presented as the mean \pm standard deviation and statistical analysis was performed using SPSS 16.0 software (SPSS, Inc., Chicago, IL, USA). Comparisons between the groups were conducted using the Student's t-test, the Mann-Whitney test or Fisher's exact method. Receiver operating characteristic (ROC) curves were used to determine the optimum threshold values for sensitivity and specificity. Correlations were analyzed using Pearson's correlation coefficient. Two-tailed P-values of $<0.05$ were considered to indicate a statistically significant difference.

\section{Results}

Preoperative and postoperative patient characteristics. A total of 92 patients, including 59 non-VAP patients and 33 VAP patients, were included in the prospective cohort study. Detailed preoperative and postoperative characteristics of the VAP and non-VAP patients are summarized in Table I. There were no statistically significant differences in preoperative characteristics between the VAP and non-VAP group patients. In addition, no statistically significant difference was observed in the occurrence of postoperative complications between the VAP and non-VAP patients $(33 / 33$ vs. $53 / 59, \mathrm{P}=0.058)$. There were six patients in the VAP group and four in the non-VAP group who suffered from multiple noninfectious postoperative complications. The SOFA scores on days 1 and 7 were significantly higher in the VAP group when compared with the non-VAP group $(\mathrm{P}<0.001)$. Furthermore, the durations of ICU stay $(\mathrm{P}=0.001)$ and mechanical ventilation $(\mathrm{P}<0.001)$ were longer in the VAP group when compared with the non-VAP group.

Serum PCT, CRP and IL-6 levels. Preoperative and postoperative (days 1, 3, 5 and 7) serum PCT, IL-6 and CRP levels were compared between patients in the VAP and non-VAP groups (Fig. 1). No statistically significant differences were observed in the preoperative serum levels of PCT, IL-6 and CRP between the VAP and non-VAP groups $(\mathrm{P}>0.05)$. However, the serum levels of PCT, IL- 6 and CRP in the VAP and non-VAP groups were significantly higher on days $1,3,5$ and 7 when compared with the preoperative baseline levels $(\mathrm{P}<0.05)$. Levels of PCT and IL-6 on day 1 were significantly higher in the VAP group when compared with the non-VAP group $(\mathrm{P}<0.05)$, while there was no statistically significant difference in the CRP levels between the groups on day $1(\mathrm{P}>0.05)$. 
Table I. Preoperative and postoperative characteristics of patients in the non-VAP and VAP groups.

\begin{tabular}{|c|c|c|c|}
\hline Characteristic & $\begin{array}{c}\text { Non-VAP } \\
\text { group }(n=59)\end{array}$ & $\begin{array}{l}\text { VAP group } \\
\quad(n=33)\end{array}$ & P-value \\
\hline${ }^{\mathrm{a} A g e}$ (years) & $47 \pm 11$ & $50 \pm 13$ & NS \\
\hline Gender, male/female (n) & $29 / 30$ & $18 / 15$ & NS \\
\hline Type of surgery (n) & & & - \\
\hline Valve surgery & 32 & 10 & - \\
\hline Coronary artery bypass grafting & 13 & 6 & - \\
\hline Ascending aortic surgery & 8 & 8 & - \\
\hline Combined surgery & 4 & 7 & - \\
\hline Others & 2 & 2 & - \\
\hline Comorbidities (n) & & & - \\
\hline Hypertension & 31 & 10 & - \\
\hline Diabetes mellitus & 10 & 4 & - \\
\hline Pulmonary diseases & 6 & 4 & - \\
\hline Kidney disease & 0 & 2 & - \\
\hline Others & 6 & 5 & - \\
\hline a'LEF (\%) & $61 \pm 13$ & $63 \pm 9$ & NS \\
\hline \multicolumn{4}{|l|}{${ }^{a}$ Baseline biological measurements } \\
\hline PCT (ng/ml) & $0.11 \pm 0.09$ & $0.20 \pm 0.21$ & NS \\
\hline IL-6 (pg/ml) & $8.44 \pm 4.32$ & $9.58 \pm 5.35$ & NS \\
\hline CRP (ng/ml) & $10.18 \pm 8.04$ & $12.33 \pm 0.76$ & NS \\
\hline${ }^{\mathrm{a}} \mathrm{CPB}$ duration (min) & $118 \pm 48$ & $156 \pm 94$ & NS \\
\hline${ }^{a}$ Aortic cross clamping time $(\mathrm{min})$ & $66 \pm 23$ & $71 \pm 41$ & NS \\
\hline${ }^{\mathrm{a}}$ Mechanical ventilation duration (days) & $3 \pm 2$ & $6 \pm 3$ & 0.000 \\
\hline aLength of stay in the ICU (days) & $8 \pm 6$ & $12 \pm 9$ & 0.001 \\
\hline Postoperative complication (n) & 53 & 33 & NS \\
\hline Cardiovascular complication (n) & 47 & 26 & - \\
\hline Pulmonary dysfunction (n) & 3 & 4 & - \\
\hline Neurological complication (n) & 0 & 2 & - \\
\hline Reoperation due to bleeding (n) & 6 & 3 & - \\
\hline AKI requiring RRT (n) & 1 & 3 & - \\
\hline aSOFA on day 1 & $9 \pm 3$ & $11 \pm 2$ & $<0.001$ \\
\hline${ }^{\mathrm{a} S O F A}$ on day 7 & $3 \pm 1$ & $7 \pm 4$ & $<0.001$ \\
\hline Mortality (n) & 3 & 7 & 0.02 \\
\hline
\end{tabular}

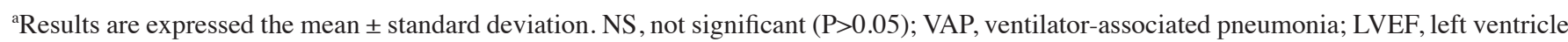
ejection fraction; PCT, procalcitonin; IL-6, interleukin-6; CRP, C-reactive protein; CPB, cardiopulmonary bypass; ICU, intensive care unit; AKI, acute kidney injury; RRT, renal replacement therapy; SOFA, sequential organ-failure assessment.

Diagnostic value of serum PCT, IL-6 and CRP levels. Using the ROC curves, it was concluded that the optimum PCT cut-off value for VAP diagnosis on day 1 was $5.0 \mathrm{ng} / \mathrm{ml}$, with a sensitivity of $91 \%$ and a specificity of $71 \%$. The areas under the ROC curve were $0.87,0.61$ and 0.56 for PCT, IL- 6 and CRP, respectively (Table II; Fig. 2).

Linear correlation analysis. Associations between the serum levels of PCT, IL- 6 and CRP in the VAP patients and the corresponding SOFA scores on days 1 and 7 were analyzed using linear correlation analysis. The PCT levels on days 1 and 7 correlated positively with the SOFA scores $(r=0.54$, $\mathrm{P}<0.001$ and $\mathrm{r}=0.66, \mathrm{P}<0.001$, respectively; Fig. 3), while the levels of IL- 6 and CRP were not found to correlate with the SOFA scores $(\mathrm{P}>0.05)$. There were no statistically significant linear correlations between the serum levels of PCT, IL-6 and CRP $(\mathrm{P}>0.05)$.

\section{Discussion}

Although numerous studies have reported the use of serum PCT as a marker for VAP (16-18), the results have been contra- 
Table II. Diagnostic value of serum levels of PCT, IL-6 and CRP on day 1.

\begin{tabular}{lcccccc}
\hline Protein & Optimal cut-off & AUC & Sensitivity (\%) & Specificity (\%) & PPV (\%) & NPV (\%) \\
\hline PCT & $5.0 \mathrm{ng} / \mathrm{ml}$ & 0.87 & 91 & 71 & 64 & 93 \\
IL-6 & $70.8 \mathrm{pg} / \mathrm{ml}$ & 0.61 & 58 & 64 & 78 \\
CRP & $108.3 \mathrm{ng} / \mathrm{ml}$ & 0.56 & 52 & 74 & 52 & 73 \\
\hline
\end{tabular}

PCT, procalcitonin; IL-6, interleukin-6; CRP, C-reactive protein; AUC, area under the receiver operating characteristic curve; PPV, positive predictive value; NPV, negative predictive value.

A

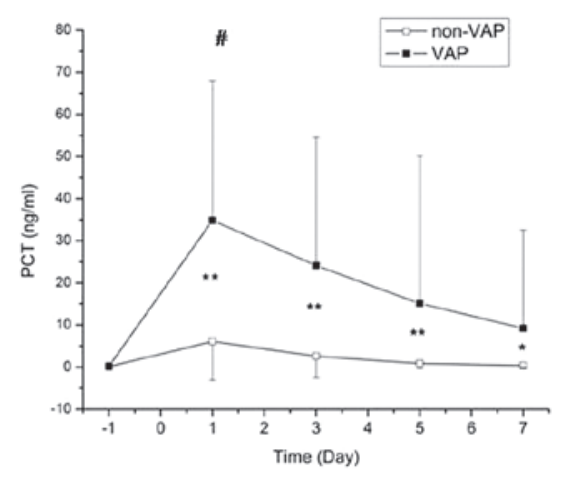

B

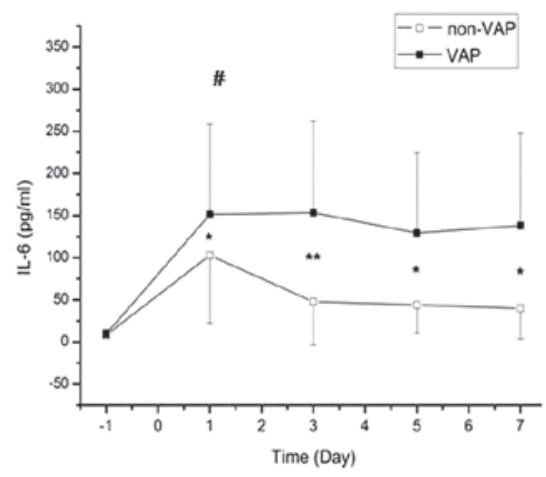

C

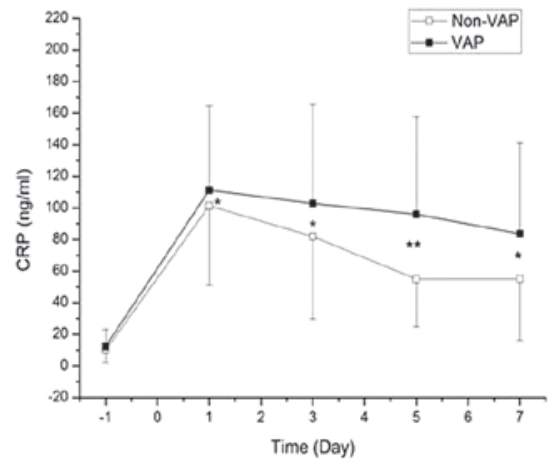

Figure 1. Comparison of the serum concentrations of (A) PCT, (B) IL-6 and (C) CRP between the VAP and non-VAP groups preoperatively and on days $1,3,5$ and 7 . ${ }^{*} \mathrm{P}<0.05$ and ${ }^{* *} \mathrm{P}<0.01$, vs. preoperative levels in both VAP and non-VAP groups; ${ }^{*} \mathrm{P}<0.05$, vs. non-VAP group on day 1 . PCT, procalcitonin; IL-6, interleukin-6; CRP, C-reactive protein; VAP, ventilator-associated pneumonia.

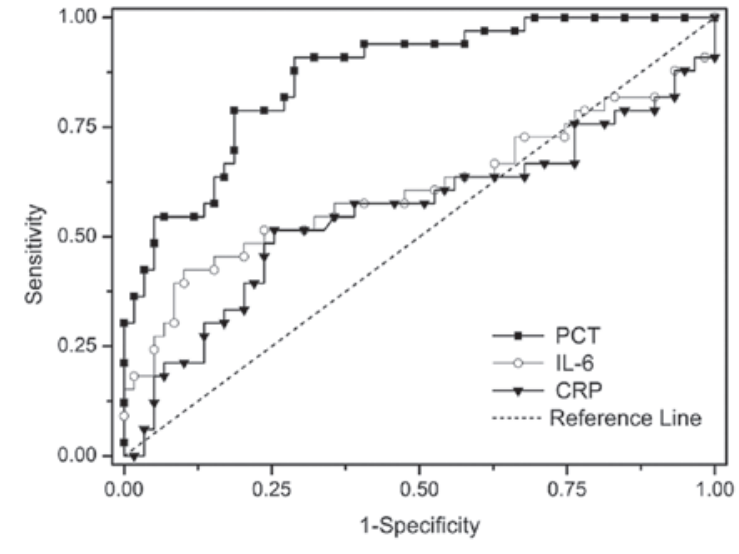

Figure 2. Receiver operating characteristic curves of serum PCT, IL-6 and CRP on day 1. PCT, procalcitonin; IL-6, interleukin-6; CRP, C-reactive protein. dictory. In the present study, the serum levels of PCT, IL-6 and CRP were measured in VAP and non-VAP cardiac surgery patients. The optimum PCT cut-off value for VAP diagnosis on day 1 was $5.0 \mathrm{ng} / \mathrm{ml}$, with a sensitivity of $91 \%$ and a specificity of $71 \%$. Furthermore, the serum levels of PCT were shown to correlate positively with the SOFA scores on days 1 and 7 . Therefore, serum PCT may be used as a diagnostic marker for VAP in patients following cardiac surgery.

Duflo et al (16) found that serum levels of PCT increased significantly in VAP patients when compared with non-VAP patients until day 3. The optimum cut-off value of serum PCT for the diagnosis of VAP was $3.9 \mathrm{ng} / \mathrm{ml}$, with a low sensitivity of $41 \%$, but an excellent specificity of $100 \%$. Oppert et al (17) studied 28 patients who exhibited a spontaneous return in circulation following cardiac arrest and determined the optimum serum PCT cut-off value to be $1 \mathrm{ng} / \mathrm{ml}$, with a 

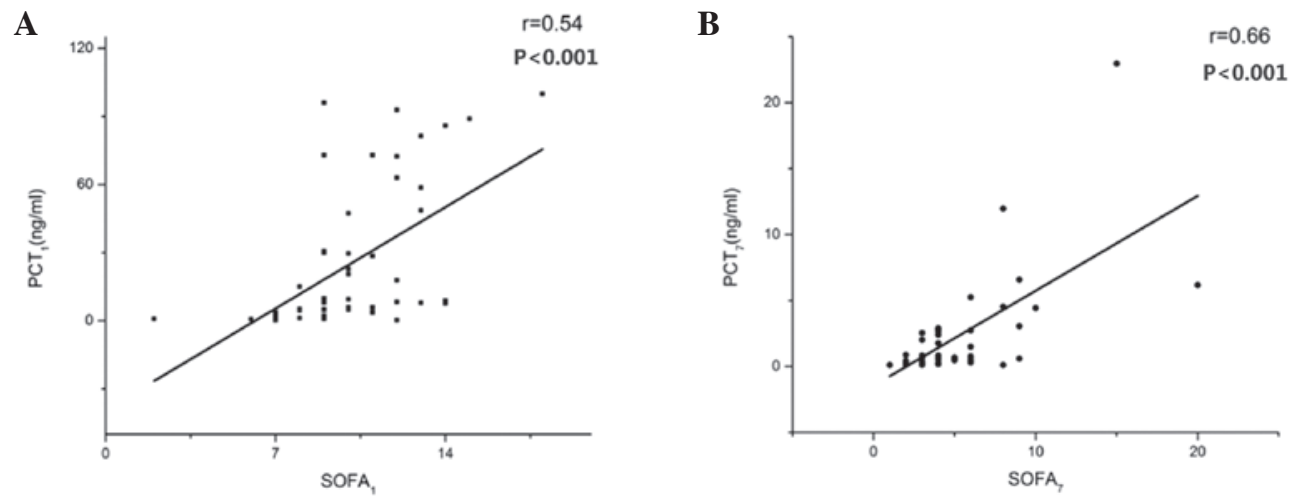

Figure 3. Linear correlation analysis between the levels of serum PCT in the VAP patients and the corresponding SOFA score on (A) day 1 and (B) day 7. PCT, procalcitonin; SOFA, sequential organ failure assessment.

sensitivity of $100 \%$ and a specificity of $75 \%$ for VAP diagnosis. However, a study of 73 suspected VAP patients by Luyt et al (18) found PCT to be a poor marker for VAP, with a sensitivity of $72 \%$ and a specificity of only $24 \%$, at a cut-off level of $0.5 \mathrm{ng} / \mathrm{ml}$. Mixed ICU, early- or late-onset of VAP and the use of antibiotics in advance of diagnosis may lead to decreased sensitivity or specificity. In the study by Luyt $\mathrm{et} \mathrm{al}$, preinfected patients were not excluded, while in the study by Oppert et al, only 10 cases among 12 patients exhibited early-onset VAP. Furthermore, the population assessed in the study by Duflo et al consisted of late-onset VAP patients who had received over two years of ventilation. These factors may have caused underestimation or overestimation of the diagnostic value of PCT, which may account for the discrepancies among previous findings. In the present study, all the patients exhibited early-onset VAP following cardiac surgery, which ensured that the subjects were selected from a homogeneous patient population.

Numerous studies have shown that cardiac surgery and CPB may affect the serum levels of various biomarkers, including PCT, CRP, IL-6 and IL-8 $(19,20)$. Among these proteins, PCT in particular is considered to be closely associated with infection conditions. In the absence of a bacterial infection, SIRS caused by surgery or CPB is an important factor in the stimulation of PCT expression (13). Cardiac surgery and CPB result in the exposure of blood vessels to nonphysiological surfaces, the translocation of endotoxin and the release of cytokines, such as IL-6, all of which have been shown to induce PCT (13). However, numerous studies (20-22) have indicated that the $\mathrm{CPB}$ procedure itself causes only a moderate, transient postoperative increase in PCT levels. PCT levels peaked on days 1 or 2 following surgery and decreased continuously to a normal value, with the peak value not generally exceeding $2 \mathrm{ng} / \mathrm{ml}$ (22). Therefore, Jebali et al suggested that the diagnostic properties of PCT may not be evident during the first two days following surgery (23). Diagnosis of VAP using PCT as a biomarker requires the patient to undergo mechanical ventilation for $>48 \mathrm{~h}$. However, all the subjects in the present study were postoperative patients who had been using a tracheal intubation for over two days; thus, the CPB-induced postoperative peak in PCT levels was avoided. Therefore, this experiment did not consider the impact of mechanical ventilation time.
Markedly increased levels of PCT are primarily associated with postoperative complications, including low cardiac output syndrome, perioperative myocardial infarction, pulmonary dysfunction, renal failure and infection (23). Prat et al (24) found that PCT levels were significantly higher in 15 patients with infectious or noninfectious complications compared with patients who recovered without complications. The optimum cut-off value of PCT on the first postoperative day was $2 \mathrm{ng} / \mathrm{ml}$, with a sensitivity of $92.3 \%$ and a specificity of $93.8 \%$. Sponholz et al (11) suggested that PCT levels were significantly higher in patients with postoperative infection when compared with those in noninfected patients. Therefore, continuous monitoring of the serum PCT levels was the most effective approach for diagnosing postoperative infection.

Although the observation period began after $>48 \mathrm{~h}$ of postoperative intubation in the present study, there remained 44/59 patients with PCT levels of $>2 \mathrm{ng} / \mathrm{ml}$ in the non-VAP group, and all the patients in the VAP group had PCT levels of $>2 \mathrm{ng} / \mathrm{ml}$ (33/33). According to the aforementioned criteria, all the patients in the VAP group exhibited noninfectious complications and there were no statistically significant differences when compared with the non-VAP group (53/59). However, the SOFA scores of the patients in the VAP group were notably higher when compared with the non-VAP group. A previous study demonstrated that higher SOFA scores were associated with significantly higher serum PCT concentrations during the course of multiple organ dysfunction syndrome (MODS) and sepsis (25). In addition, Haasper et al (26) showed that the presence of significantly higher levels of PCT in patients resulted in the development of MODS. Differences in SOFA scores may increase the levels of PCT in the VAP group to a greater extent compared with the non-VAP group, which may indirectly increase the diagnostic accuracy of VAP. In the present study, the postoperative PCT levels in the VAP group were significantly higher when compared with the non-VAP group during the observation period. PCT levels of $>5 \mathrm{ng} / \mathrm{ml}$ had a sensitivity of $91.2 \%$ and a specificity of $82.4 \%$, respectively. A study by Ramirez et al (27) examined 44 patients who received mechanical ventilation for $>48 \mathrm{~h}$ in a medical ICU, of which 20 patients were suspected of having VAP. Based on a pathological examination, nine individuals were diagnosed with VAP, while 11 patients were 
assigned to the non-VAP group. On the day of VAP diagnosis confirmation, serum PCT levels and SOFA scores in the VAP group were significantly higher compared with those in the non-VAP group. The optimum cut-off value of PCT was $2.99 \mathrm{ng} / \mathrm{ml}$, with a sensitivity of $78 \%$ and a specificity of 97\%. The optimum serum PCT cut-off value in the present study was higher $(5 \mathrm{ng} / \mathrm{ml})$ when compared with the results of Oppert et al (17) and Ramirez et al (27), which may be due to the recruitment of patients from the cardiac surgery ICU, exhibiting more serious conditions and postoperative complications and higher SOFA scores.

According to a previous study, IL-6 is a cytokine secreted by giant eosinophilic cells that participates in the acute inflammatory process (28). IL-6 levels are known to increase in cardiac surgery and may be involved in the postoperative cytokine cascade (29). CRP synthesized in the liver is a nonspecific biological marker for inflammation (30). Jebali et al showed that serum IL-6 was not a marker for postoperative complications following cardiac surgery (23). However, the use of CRP for the diagnosis of VAP remains controversial. Póvoa et al (30) showed that a CRP level of $>960 \mathrm{mg} / \mathrm{l}$ provided a good accuracy for VAP diagnosis, with a sensitivity of $87 \%$ and a specificity of $88 \%$. In the present study, serum levels of CRP in the VAP and non-VAP groups did not show a statistically significant difference until three days following the diagnosis of VAP, and CRP exhibited no diagnostic value on day 1 . The optimum cut-off value of IL-6 for VAP diagnosis was $70.8 \mathrm{pg} / \mathrm{ml}$; however, the diagnostic accuracy was relatively low, with a sensitivity of $58 \%$ and a specificity of 64\%. These results indicated that IL-6 and CRP were not effective for the diagnosis of VAP following cardiac surgery with CPB. Although IL-6 has been demonstrated to induce the production of PCT in vitro (26), no correlation was found between the levels of IL-6 and PCT.

There were a number of limitations to the present study. Firstly, the sample size was relatively small, which may have affected the diagnostic capability of PCT. Thus, further studies with a larger sample size are required to verify the results. Secondly, the present study examined only patients with early-onset VAP who had undergone elective cardiac surgery. Therefore, the results may differ from other studies that included early-onset VAP patients who were recruited from a noncardiac surgical ICU or cardiac surgery patients with late-onset VAP. Finally, the effects of surgery and CPB on serum levels of PCT remain unknown, as it was not possible to obtain the premorbid serum PCT concentrations of the patients.

In conclusion, PCT may play an important role as a biomarker in the diagnosis of early-onset VAP following cardiac surgery in clinical practice. Furthermore, serum PCT levels and SOFA scores were shown to positively correlate. However, these results require validation by further studies.

\section{References}

1. Hortal J, Giannella M, Pérez MJ, et al: Incidence and risk factors for ventilator-associated pneumonia after major heart surgery. Intensive Care Med 35: 1518-1525, 2009.

2. Vijarnsorn C, Winijkul G, Laohaprasitiporn D, et al: Postoperative fever and major infections after pediatric cardiac surgery. J Med Assoc Thai 95: 761-770, 2012.
3. Melsen WG, Rovers MM, Groenwold RH, et al: Attributable mortality of ventilator-associated pneumonia: a meta-analysis of individual patient data from randomised prevention studies. Lancet Infect Dis 13: 665-671, 2013.

4. Hortal J, Muñoz P, Cuerpo G, Litvan H, Rosseel PM and Bouza E; European Study Group on Nosocomial Infections; European Workgroup of Cardiothoracic Intensivists: Ventilator-associated pneumonia in patients undergoing major heart surgery: an incidence study in Europe. Crit Care 13: R80, 2009.

5. Roeleveld PP, Guijt D, Kuijper EJ, Hazekamp MG, de Wilde RB and de Jonge E: Ventilator-associated pneumonia in children after cardiac surgery in The Netherlands. Intensive Care Med 37: 1656-1663, 2011.

6. Tamayo E, Álvarez FJ, Martínez-Rafael B, et al; Valladolid Sepsis Study Group: Ventilator-associated pneumonia is an important risk factor for mortality after major cardiac surgery. J Crit Care 27: 18-25, 2012

7. Becker KL, Snider R and Nylen ES: Procalcitonin assay in systemic inflammation, infection, and sepsis: clinical utility and limitations. Crit Care Med 36: 941-952, 2008.

8. Assicot M, Gendrel D, Carsin H, Raymond J, Guilbaud J and Bohuon C: High serum procalcitonin concentrations in patients with sepsis and infection. Lancet 341: 515-518, 1993.

9. Riedel S: Procalcitonin and the role of biomarkers in the diagnosis and management of sepsis. Diagn Microbiol Infect Dis 73: 221-227, 2012.

10. Rea-Neto A, Youssef NC, Tuche F, et al: Diagnosis of ventilator-associated pneumonia: a systematic review of the literature. Crit Care 12: R56, 2008.

11. Sponholz C, Sakr Y, Reinhart K and Brunkhorst F: Diagnostic value and prognostic implications of serum procalcitonin after cardiac surgery: a systematic review of the literature. Crit Care 10: R145, 2006.

12. Adib-Conquy M, Monchi M, Goulenok C, et al: Increased plasma levels of soluble triggering receptor expressed on myeloid cells 1 and procalcitonin after cardiac surgery and cardiac arrest without infection. Shock 28: 406-410, 2007.

13. Sinning JM, Scheer AC, Adenauer V, et al: Systemic inflammatory response syndrome predicts increased mortality in patients after transcatheter aortic valve implantation. Eur Heart J 33: 1459-1468, 2012.

14. American Thoracic Society; Infectious Diseases Society of America: Guidelines for the management of adults with hospital-acquired, ventilator-associated, and healthcare-associated pneumonia. Am J Respir Crit Care Med 171: 388-416, 2005.

15. Vincent JL, Moreno R, Takala J, et al: The SOFA (Sepsis-related Organ Failure Assessment) score to describe organ dysfunction/failure. On behalf of the Working Group on Sepsis-Related Problems of the European Society of Intensive Care Medicine. Intensive Care Med 22: 707-710, 1996.

16. Duflo F, Debon R, Monneret G, Bienvenu J, Chassard D and Allaouchiche B: Alveolar and serum procalcitonin: diagnostic and prognostic value in ventilator-associated pneumonia. Anesthesiology 96: 74-79, 2002.

17. Oppert M, Reinicke A, Müller C, Barckow D, Freix U and Eckardt KU: Elevations in procalcitonin but not C-reactive protein are associated with pneumonia after cardiopulmonary resuscitation. Resuscitation 53: 167-170, 2002.

18. Luyt CE, Combes A, Reynaud C, et al: Usefulness of procalcitonin for the diagnosis of ventilator-associated pneumonia. Intensive Care Med 34: 1434-1440, 2008.

19. Hensel M, Volk T, Döcke WD, et al: Hyperprocalcitonemia in patients with noninfectious SIRS and pulmonary dysfunction associated with cardiopulmonary bypass. Anesthesiology 89: 93-104, 1998.

20. Amin DN, Pruitt JC and Schuetz P: Influence of major cardiopulmonary surgery on serum levels of procalcitonin and other inflammatory markers. Anaesth Intensive Care 40: 760-766, 2012.

21. Beghetti M, Rimensberger PC, Kalangos A, Habre W and Gervaix A: Kinetics of procalcitonin, interleukin 6 and C-reactive protein after cardiopulmonary-bypass in children. Cardiol Young 13: 161-167, 2003.

22. Meisner M, Rauschmayer C, Schmidt J, et al: Early increase of procalcitonin after cardiovascular surgery in patients with postoperative complications. Intensive Care Med 28: 1094-1102, 2002.

23. Jebali MA, Hausfater P, Abbes Z, Aouni Z, Riou B and Ferjani M: Assessment of the accuracy of procalcitonin to diagnose postoperative infection after cardiac surgery. Anesthesiology 107: 232-238, 2007. 
24. Prat C, Ricart P, Ruyra X, et al: Serum concentrations of procalcitonin after cardiac surgery. J Card Surg 23: 627-632, 2008.

25. Meisner M, Tschaikowsky K, Palmaers T and Schmidt J: Comparison of procalcitonin (PCT) and C-reactive protein (CRP) plasma concentrations at different SOFA scores during the course of sepsis and MODS. Crit Care 3: 45-50, 1999.

26. Haasper C, Kalmbach M, Dikos GD, et al: Prognostic value of procalcitonin (PCT) and/or interleukin-6 (IL-6) plasma levels after multiple trauma for the development of multi organ dysfunction syndrome (MODS) or sepsis. Technol Health Care 18: 89-100, 2010.
27. Ramirez P, Garcia MA, Ferrer M, et al: Sequential measurements of procalcitonin levels in diagnosing ventilator-associated pneumonia. Eur Respir J 31: 356-362, 2008.

28. Hammer S, Fuchs AT, Rinker C, et al: Interleukin-6 and procalcitonin in serum of children undergoing cardiac surgery with cardiopulmonary bypass. Acta Cardiol 59: 624-629, 2004.

29. Denizot Y, Lorgeot V, Cornu E and Nathan N: Plasma leukaemia inhibitory factor, interleukin 6 and soluble interleukin 6 receptor levels during cardiopulmonary bypass with extracorporeal circulation. Cytokine 10: 303-306, 1998.

30. Póvoa P: C-reactive protein: a valuable marker of sepsis. Intensive Care Med 28: 235-243, 2002. 